\title{
Safety and preliminary signs of efficacy with the combination of pembrolizumab plus oxaliplatin and S-1 in Japanese gastric cancer patients
}

\author{
Maria Alsina ${ }^{1}$, Elizabeth C. Smyth ${ }^{2}$ \\ ${ }^{1}$ Department of Medical Oncology Vall d'Hebron University Hospital and VHIO Vall d'Hebron Insitute of Oncology, Barcelona, Spain; \\ ${ }^{2}$ Department of Oncology, Cambridge University Hospitals NHS Foundation Trust, Cambridge, UK \\ Correspondence to: Maria Alsina. Department of Medical Oncology Vall d'Hebron University Hospital and VHIO Vall d'Hebron Insitute of Oncology, \\ Passeig de la Vall d'Hebron 119-129, 08035, Barcelona, Spain. Email: malsina@vhio.net. \\ Comment on: Kawazoe A, Yamaguchi K, Yasui H, et al. Safety and efficacy of pembrolizumab in combination with S-1 plus oxaliplatin as a first-line \\ treatment in patients with advanced gastric/gastroesophageal junction cancer: Cohort 1 data from the KEYNOTE-659 phase IIb study. Eur J Cancer \\ 2020;129:97-106.
}

Submitted Jun 16, 2020. Accepted for publication Jul 01, 2020.

doi: $10.21037 /$ atm-20-4725

View this article at: http://dx.doi.org/10.21037/atm-20-4725

Gastric and gastroesophageal junction cancer (GC) represents a world-wide problem due to its high prevalence and aggressive nature. Advanced GC patients have an extremely poor prognosis with median overall survival (OS) of approximately 10 months (1). Many molecular targeted agents have been evaluated in conjunction with first-line chemotherapy treatment, however none except trastuzumab has demonstrated significant efficacy (2). Immune checkpoint inhibitors (ICI) targeting programmed death-1 (PD-1) and PD-L1 have shown preliminary good results in chemorefractory GC, although have sometimes been disappointing in earlier disease stages (Table 1) (3).

The KEYNOTE-659 phase IIb study was a nonrandomized, multicenter, open-label phase IIb study which evaluated the safety and efficacy of pembrolizumab added to first-line chemotherapy in Japanese GC patients. Eligible patients were programmed death-1 ligand (PD-L1) positive and human epidermal growth factor receptor 2 (HER2)negative. Kawazoe et al. (4) have recently reported the results KEYNOTE-659 cohort 1, which evaluated the combination of pembrolizumab with S-1 plus oxaliplatin (SOX). The results presented by Kawazoe et al. (4) demonstrate the safety and preliminary effectiveness required to further explore the combination of pembrolizumab in combination with firstline SOX chemotherapy.

The history of GC includes multiple randomized clinical trials evaluating targeted agents and ICIs that have been negative, mainly due to a lack of a biomarker selection and the intrinsic heterogeneity of this tumor (5). Except for the demonstrated superiority of nivolumab against placebo in chemorefractory Asian GC patients (6), there is an inconsistency of the benefit offered by the ICIs in an unselected GC population. Fortunately, comprehensive analysis of these data relating to efficacy of ICI in GC highlights some biomarkers of response, linked to the tumor cell (MSI status, tumor mutational burden) and to the stroma (Epstein-Barr virus subtype and PD-L1 expression) (7). Moreover, some clinical characteristics of the patient are probably associated with the response (8).

MSI status is today the most solid biomarker, with a consistent positive correlation demonstrated in all the treatment lines, with both anti-PD1 and anti-PD-L1 agents $(3,8-10)$. A second moderately robust biomarker in GC is $\mathrm{PD}-\mathrm{L} 1$ status assessed by the combined positive score (CPS). With regard to CPS score, a higher cutoff level better correlates with a significant immune response (3). In terms of clinical biomarkers, and taking into consideration the fragility of GC patients and the relatively long time that is required for the activation of the immune response, patients with a better ECOG status and smaller volume disease are more likely to derive a greater benefit from the ICI therapy $(3,8)$. Furthermore, the activity of the ICIs is improved if given early in the tumor life (in early lines), in order to avoid lymphocyte exhaustion $(11,12)$. And finally, the ethnicity 
Table 1 Immune checkpoint inhibitors in combination with a first chemotherapy line in gastric cancer

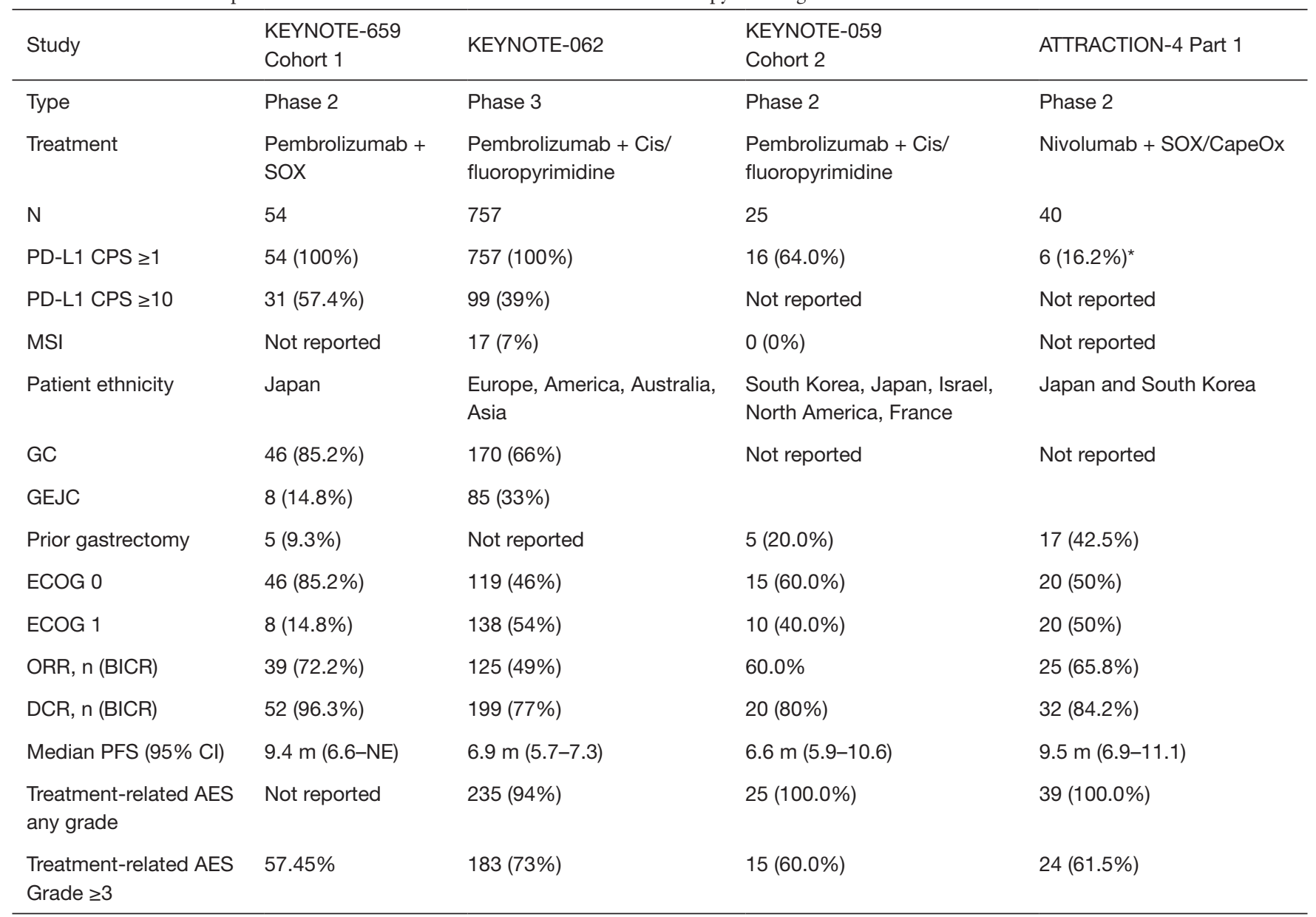

*, Tumor expression. AES, adverse events; BICR, blinded independent central review; CapeOX, capecitabine plus oxaliplatin; Cis, cisplatin; CPS, combined positive score; DCR, disease control rate; ECOG PS, Eastern Cooperative Oncology Group performance status; GEJC, gastro-esophageal junction cancer; GC, gastric cancer; m, months; MSI, microsatellite-instability; N, number of patients; ORR, overall response rate; PD-L1, programmed death ligand 1; SOX, S-1 (tegafur-gimeracil-oteracil potassium) plus oxaliplatin.

of patient also seems to matter, as Asian patients appear to benefit more than patients from other regions of the world (3). Whether Asian patients have predominantly gastric cancers, Western patients have more junctional tumors. Although the underlying biology is shared (13), Asian and non-GC present different immune-related components which may influence in the clinical outcome (14).

In the Cohort 1 of the KEYNOTE-659 study, fifty-four Japanese GC patients were treated with pembrolizumab plus SOX as a first line of treatment. Notably, the patients included in this trial had characteristics associated with a good immune response. All of them (100\%) were Japanese, 46 (85.2\%) had ECOG 0, 49 (90.7\%) had a previous gastrectomy, and $31(57.4 \%)$ had PD-L1 CPS $\geq 10$. Unfortunately, MSI status was not tested. The schema of the treatment was almost equal to the one of the KEYNOTE-062 study except for the chemotherapy backbone, which was SOX in this trial (versus cisplatin and fluoropyrimidine in KEYNOTE-062). The combination of SOX and pembrolizumab is safe; toxicity is in line with the toxicity reported in other similar clinical trials (Table 1). The primary endpoint was ORR assessed by blinded independent central review (BICR), which was $72.7 \%$. Median progression-free survival (PFS) was 9.4 months (95\% CI: 6.6-NE) and OS was not-reached.

Until now, three phase 2 studies [Cohort 1 of the KEYNOTE-659 (4), Cohort 2 of the KEYNOTE-059 (12), and Part 1 of the ATTRACTION-4 (15)] and one phase 3 study [KEYNOTE-062 (3)] have reported the efficacy of the addition of an anti-PD1 treatment to the first 
chemotherapy line in GC patients (Table 1). The ORR reported by Kawazoe et al. (4) is the best of the four trials, as well as the other surrogate end-points. These results are encouraging, but one may consider some factors that should contributed to these findings: first, it is a phase II study with good selection of patients (ECOG 0, high PD-L1 expression, Asian ethnicity); and second, the chemotherapy backbone oxaliplatin is a better partner for pembrolizumab due to its putative effect on induced immunogenic-cell death (16).

In summary, with only 54 patients included, cohort 1 of the KEYNOTE-659 study validates the safety of the combination of pembrolizumab with a first-line SOX chemotherapy treatment in GC patients. A definitive answer on efficacy of this combination would need to wait for further validation in a phase 3 randomized control trial. That said, a recent regulatory filing for nivolumab, another PD-1 inhibitor, in conjunction with SOX chemotherapy in Japan suggests that the part 2 of the ATTRACTION-4 study, which randomize Japanese naïve GC patients to SOX chemotherapy \pm nivolumab, is likely to have a positive outcome. If so, Japanese GC patients may not need to wait much longer to integrate ICI into earlier lines of treatment.

\section{Acknowledgments}

Funding: None.

\section{Footnote}

Provenance and Peer Review: This article was commissioned by the editorial office, Annals of Translational Medicine. The article did not undergo external peer review.

Conflicts of Interest: Both authors have completed the ICMJE uniform disclosure form (available at http://dx.doi. org/10.21037/atm-20-4725). MA reports personal fees from MSD, personal fees from BMS, personal fees from Servier, personal fees from Lilly, outside the submitted work. ECS reports personal fees from Astellas, personal fees from Astrazeneca, personal fees from BMS, personal fees from Celgene, personal fees from Five Prime Therapeutics, personal fees from Gritstone Oncology, personal fees from Merck, personal fees from Servier, personal fees from Zymeworks, outside the submitted work.

Ethical Statement: The authors are accountable for all aspects of the work in ensuring that questions related to the accuracy or integrity of any part of the work are appropriately investigated and resolved.

Open Access Statement: This is an Open Access article distributed in accordance with the Creative Commons Attribution-NonCommercial-NoDerivs 4.0 International License (CC BY-NC-ND 4.0), which permits the noncommercial replication and distribution of the article with the strict proviso that no changes or edits are made and the original work is properly cited (including links to both the formal publication through the relevant DOI and the license). See: https://creativecommons.org/licenses/by-nc-nd/4.0/.

\section{References}

1. Wagner AD, Unverzagt S, Grothe W, et al. Chemotherapy for advanced gastric cancer. Cochrane Database Syst Rev 2010;(3):CD004064.

2. Bang Y-J, Van Cutsem E, Feyereislova A, et al. Trastuzumab in combination with chemotherapy versus chemotherapy alone for treatment of HER2-positive advanced gastric or gastro-oesophageal junction cancer (ToGA): a phase 3, open-label, randomised controlled trial. Lancet 2010;376:687-97.

3. Tabernero J, Van Cutsem E, Bang YJ, et al. Pembrolizumab with or without chemotherapy versus chemotherapy for advanced gastric or gastroesophageal junction (G/GEJ) adenocarcinoma: The phase III KEYNOTE-062 study. J Clin Oncol 2019;37. DOI: 10.1200/JCO.2019.37.18_ suppl.LBA4007.

4. Kawazoe A, Yamaguchi K, Yasui H, et al. Safety and efficacy of pembrolizumab in combination with S-1 plus oxaliplatin as a first-line treatment in patients with advanced gastric/gastroesophageal junction cancer: Cohort 1 data from the KEYNOTE-659 phase IIb study. Eur J Cancer 2020;129:97-106.

5. Alsina M, Miquel JM, Diez M, et al. How I treat gastric adenocarcinoma. ESMO Open 2019;4:e000521.

6. Kang Y-K, Boku N, Satoh T, et al. Nivolumab in patients with advanced gastric or gastro-oesophageal junction cancer refractory to, or intolerant of, at least two previous chemotherapy regimens (ONO-4538-12, ATTRACTION-2): a randomised, double-blind, placebocontrolled, phase 3 trial. Lancet 2017;390:2461-71.

7. Cristescu R, Lee J, Nebozhyn M, et al. Molecular analysis of gastric cancer identifies subtypes associated with distinct clinical outcomes. Nat Med 2015;21:449-56.

8. Shitara K, Özgüroğlu M, Bang Y-J, et al. Pembrolizumab 
versus paclitaxel for previously treated, advanced gastric or gastro-oesophageal junction cancer (KEYNOTE-061): a randomised, open-label, controlled, phase 3 trial. Lancet 2018;392:123-33.

9. Muro K, Chung HC, Shankaran V, et al. Pembrolizumab for patients with PD-L1-positive advanced gastric cancer (KEYNOTE-012): a multicentre, open-label, phase 1b trial. Lancet Oncol 2016;17:717-26.

10. Moehler MH, Dvorkin M, Ozguroglu M, et al. Results of the JAVELIN Gastric 100 phase 3 trial: avelumab maintenance following first-line (1L) chemotherapy (CTx) vs continuation of CTx for HER2 - advanced gastric or gastroesophageal junction cancer (GC/GEJC). J Clin Oncol 2020;38:278.

11. Fuchs CS, Doi T, Jang RW, et al. Safety and efficacy of pembrolizumab monotherapy in patients with previously treated advanced gastric and gastroesophageal junction cancer: phase 2 clinical KEYNOTE-059 trial. JAMA Oncol 2018;4:e180013.

12. Bang YJ, Kang YK, Catenacci DV, et al. Pembrolizumab alone or in combination with chemotherapy as first-

Cite this article as: Alsina M, Smyth EC. Safety and preliminary signs of efficacy with the combination of pembrolizumab plus oxaliplatin and S-1 in Japanese gastric cancer patients. Ann Transl Med 2020;8(24):1696. doi: 10.21037/atm-20-4725 line therapy for patients with advanced gastric or gastroesophageal junction adenocarcinoma: results from the phase II nonrandomized KEYNOTE-059 study. Gastric Cancer 2019;22:828-37.

13. Network CGAR. Comprehensive molecular characterization of gastric adenocarcinoma. Nature 2014;513:202-9.

14. Lin SJ, Gagnon-Bartsch JA, Tan IB, et al. Signatures of tumour immunity distinguish Asian and non-Asian gastric adenocarcinomas. Gut 2015;64:1721-31.

15. Boku N, Ryu M-H, Kato K, et al. Safety and efficacy of nivolumab in combination with $\mathrm{S}-1 /$ capecitabine plus oxaliplatin in patients with previously untreated, unresectable, advanced, or recurrent gastric/ gastroesophageal junction cancer: interim results of a randomized, phase II trial (ATTRACTION-4). Ann Oncol 2019;30:250-8.

16. Tesniere A, Schlemmer F, Boige V, et al. Immunogenic death of colon cancer cells treated with oxaliplatin. Oncogene 2010;29:482-91. 